\title{
Polarization Impact in TanDEM-X Data Over Vertical-Oriented Vegetation: The Paddy-Rice Case Study
}

\author{
Esra Erten, Cristian Rossi, and Onur Yüzügüllü
}

\begin{abstract}
It has been recently shown that the TanDEM-X mission is capable of tracking the plant growth of rice paddies. The precision of the elevation measure depends on the physical interaction between the synthetic aperture radar (SAR) signal and the canopy. In this letter, this interaction is studied by considering the signal polarization. In particular, the vertical and horizontal wave polarizations are compared, and their performance in the temporal mapping of the crop height is analyzed. The temporal elevation difference analysis shows a monotonically increasing trend within the reproductive stage of the canopy, with maximum height discrepancies between polarizations of about $9 \mathrm{~cm}$. From an operational point of view of InSAR-based vegetation height measurements, this letter demonstrates that the oriented structure of the canopy shall be considered not only in polarimetric InSAR studies but also in the interpretation of bistatic spaceborne interferometric elevation models.
\end{abstract}

Index Terms-Agriculture, copolar phase difference (CPD), polarimetry, synthetic aperture radar (SAR), TanDEM-X, X-Band.

\section{INTRODUCTION}

$\mathbf{T}$ HE canopy height is a primary structural characteristic for phenological stage studies, carbon storage, and biomass calculations. Because of its importance, canopy height estimation has been studied many times by making use of remote sensing technologies, particularly synthetic aperture radar (SAR). Related works can be gathered into two groups.

First, a considerable amount of research has been done by using radiative transfer theory (RTV). RTV is applied to calculate the radar backscattering that depends on the geometric and physical features of the canopy [1]-[3]. These studies indicate that backscattering is based on the canopy's physical attributes such as the leaf area index, the plant height, the stem width, the number of leaves, the leaf angle, and the leaf size. Due to the complicated electromagnetic interaction between radar

Manuscript received December 16, 2014; revised February 18, 2015; accepted March 3, 2015. This work was supported by the Scientific and Technological Research Council of Turkey (TUBITAK) under Project 113 Y446.

E. Erten is with the Department of Geomatics Engineering, Istanbul Technical University, 34469 Istanbul, Turkey.

C. Rossi is with the Remote Sensing Technology Institute, German Aerospace Center, 82234 Wessling, Germany.

O. Yüzügüllü is with the Institute of Environmental Engineering, Swiss Federal Institute of Technology (ETH) Zurich, 8093 Zurich, Switzerland.

Color versions of one or more of the figures in this paper are available online at http://ieeexplore.ieee.org.

Digital Object Identifier 10.1109/LGRS.2015.2410339 waves and the vegetation canopy, it is not easy to develop the direct relationship of the backscattering with biophysical parameters. Thus, for estimating the canopy's physical parameters from SAR backscattering values, Monte Carlo simulations are required [2].

Second, interferometric phase information, which is a direct function of the canopy height, i.e., not an indirect function as backscattering information, provides an additional instrument to canopy studies with the sole cost of two SAR images [4], [5]. From an agricultural application's point of view, the interferometric phase for the operational canopy height calculation requires a particular system configuration. Since the plant is temporally growing, two acquisitions at different dates will provide very low coherence and no stable phase information. To overcome this limit, the acquisitions must be commanded at the same time. Among them, the TanDEM-X is the first spaceborne mission designed to globally acquire data in order to generate digital elevation models (DEMs) at High-Resolution Terrain Information 3 (HRTI-3) standards [6], [7].

An analysis of TanDEM-X capabilities in assessing canopy heights was performed in [8]. This study shows that TanDEM$\mathrm{X}$ images have a great potential in mapping the canopy height through almost the entire plant growth. A relevant feature is the high resolution of the sensors, which brings the assessment on the field level. However, the strong variability in the location of the phase center through the canopy causes underestimations for the estimated height. This depends on the phenology of the crops and the system parameters (polarization, bandwidth, etc.). The objective of this study is to deeply analyze the capabilities and limitations of TanDEM-X monitoring by considering the wave polarization. The work in [8] showed the relationship between the crop height and the differential interferometric phase for paddy rice using the $\mathrm{HH}$ polarimetric channel. In this letter, the VV channel is also considered, and a comprehensive comparison between the two polarizations is presented. Observed height differences are theoretically related to the path of the wave through the canopy, which has to be considered when plants are in the reproductive stage. Recent work [9] has also studied the polarization-dependent height differences in TanDEM-X acquisitions for the assessment of snow depth.

This letter is organized as follows. In Section II, the study area and the data set are introduced. Additionally, the phenology of the crops on the acquisition dates is briefly described by making use of polarimetric features. Section III analyzes the estimated crop heights for both polarizations. Section IV concludes this letter with the main outcomes. 


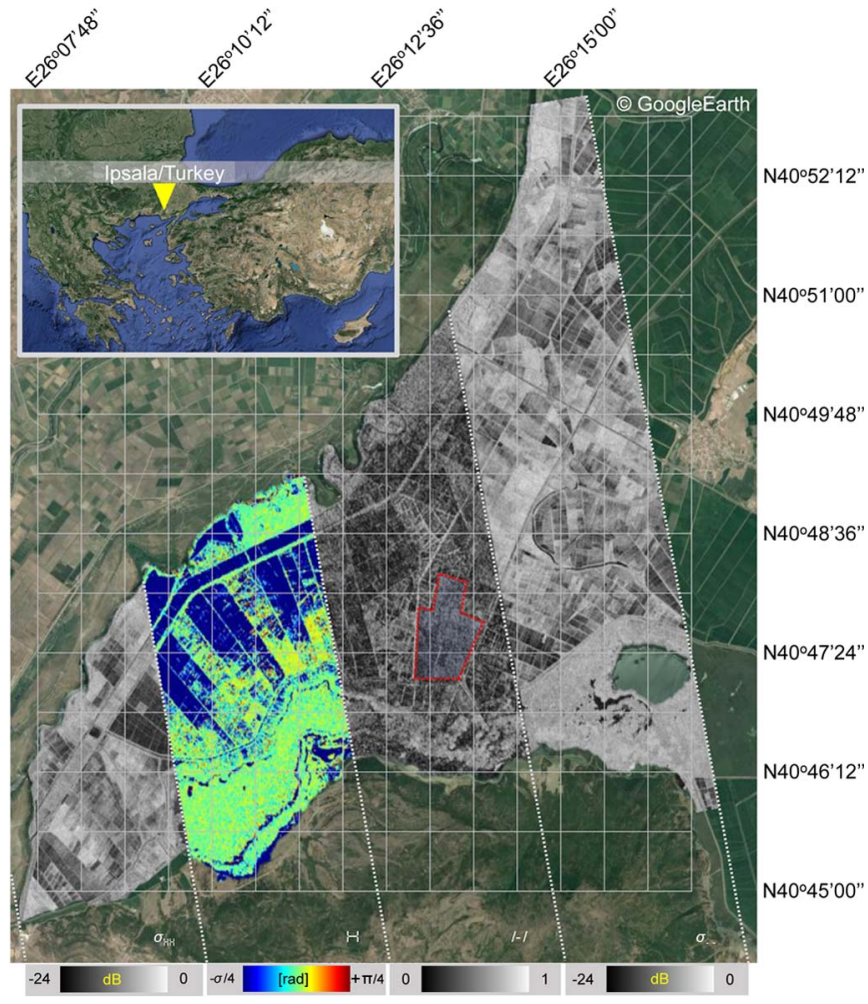

Fig. 1. Agricultural study area in Ipsala, Turkey. The copolar images acquired on June 21, 2014 in the HH and VV polarizations, and their coherence and phase difference measurements are placed over the Google Earth image. The selected fields for the study are highlighted by the red dashed line.

\section{Study AREA And COMPlex IMAGe AnAlysis}

The study area is a unique wetland environment consisting of lakes, rivers, and agricultural rice fields, which are visually detectable on the Google Earth image given in Fig. 1. In this region, which is located in Ipsala, Turkey, the agricultural practices of paddies are owner dependent, i.e., with time shifts between seeding days among neighbor agricultural fields. Nevertheless, fields are generally flooded and seeded during late May and are harvested in early October. In the study area, the sowing method is direct seeding by broadcasting, implying random seeding instead of regular straight-row seeding. To give an overview of a spatial distribution and heterogeneity in the sowing procedure of the agricultural fields, the backscattering images acquired on June 21, 2014 in the HH and VV polarizations, and their coherence and phase difference measurements are placed over the Google Earth image in Fig. 1. Differences in polarimetric observations among the agricultural fields can be detected.

Interferometric SAR (InSAR)-based vegetation height measurements were analyzed using a stack of nine $\mathrm{HH}$ and $\mathrm{VV}$ dual-polarization TanDEM-X images acquired through the cultivation period of 2012 , with an incidence angle of $37^{\circ}$. Some relevant properties of the images are given in Fig. 2. For each observation point, the vertical error bar indicates the height of ambiguity that is inversely proportional to the baseline, whereas the horizontal error bar shows the unreliability of the $\mathrm{HH}$ interferometric observations with the equality $1-|\gamma|$, where $|\gamma|$ represents the mean coherence value. These coherence values are obtained by averaging the estimates over the 50 neighbor

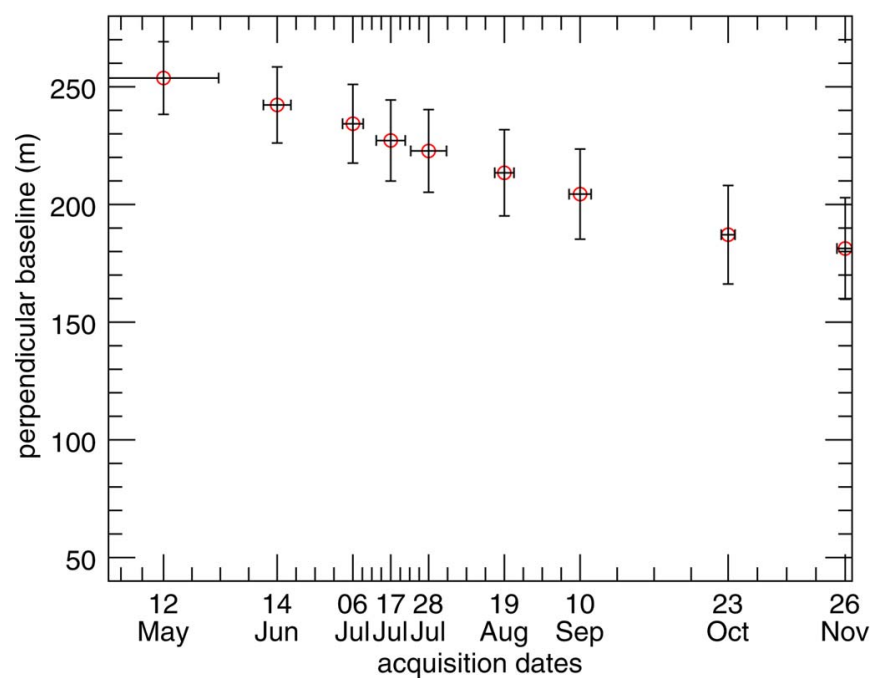

Fig. 2. Perpendicular baselines of TanDEM-X and TerraSAR-X acquisitions with respect to the acquisition dates. For each acquisition date, the vertical error bar indicates the height of ambiguity, whereas the horizontal error bar shows the unreliability of the $\mathrm{HH}$ interferometric acquisitions due to the coherence values, i.e., the smaller $1-|\gamma|$ is, the better accuracy is achieved.

agricultural fields highlighted in Fig. 1, which are analyzed in the following sections.

Once coherence maps, interferograms, and DEMs have been generated with the interferometric TanDEM-X processor [7], the next step is to identify the phenological stages of the canopy during the acquisitions. Here, it is necessary to remind that this letter contains a concise presentation of the height measurement differences based on phenological stages and polarimetric acquisitions. It is not intended to give an in-depth description of the bistatic interferometric processing but merely the characterization of elevation measurements considering the polarization (see [7] and [8] for detailed information about the process of a bistatic interferometric configuration).

To identify the phenological stages of the fields, polarimetric features such as copolar backscattering values and their ratio, the copolar phase difference (CPD), and entropy are analyzed among the 50 selected agricultural fields. First, four polarimetric features, which are obtained from the polarimetric acquisition vector $k=\left[S_{\mathrm{HH}} S_{\mathrm{vV}}\right]$ for the whole canopy, are expressed by the following:

$$
\begin{aligned}
& \sigma_{\mathrm{HH}}=10 \log _{10}\left\langle\left|S_{\mathrm{HH}} S_{\mathrm{HH}}^{*}\right|\right\rangle \quad[\mathrm{dB}] \\
& \sigma_{\mathrm{vV}}=10 \log _{10}\left\langle\left|S_{\mathrm{vV}} S_{\mathrm{vV}}^{*}\right|\right\rangle \\
& \varphi=\tan ^{-1}\left(\frac{\left\langle S_{\mathrm{HH}} S_{\mathrm{vV}}^{*}\right\rangle}{\sqrt{\left\langle\left|S_{\mathrm{HH}}\right|^{2}\right\rangle\left\langle\left|S_{\mathrm{VV}}\right|^{2}\right\rangle}}\right) \quad[\mathrm{rad}] \\
& R_{\mathrm{HH}, \mathrm{VV}}=10 \log _{10}\left(\frac{\left\langle\left|S_{\mathrm{HH}}\right|^{2}\right\rangle}{\left\langle\left|S_{\mathrm{VV}}\right|^{2}\right\rangle}\right)[\mathrm{dB}]
\end{aligned}
$$

where $\sigma_{\mathrm{HH}}$ and $\sigma_{\mathrm{VV}}$ are the backscattering coefficients for the $\mathrm{HH}$ and VV channels, respectively, $\varphi$ is the CPD, and $R_{\mathrm{HH}, \mathrm{VV}}$ is the backscattering ratio. Fig. 3 shows the plots of the polarimetric features for all of the monitored fields, in which each field is shown by a different color. To tackle the heterogeneity inside the fields, $K$-means clustering was applied before assigning a value for each field, as detailed in [10]. 


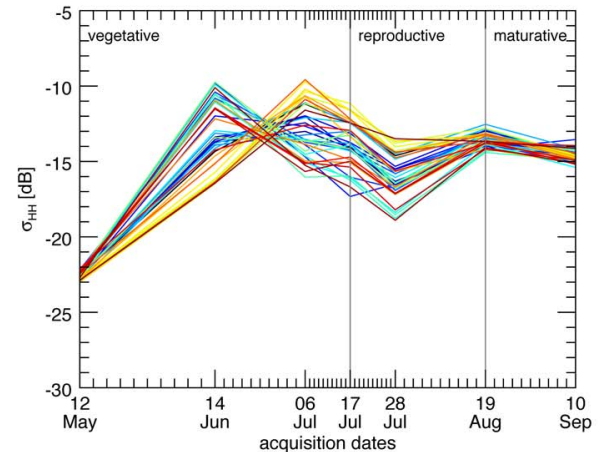

(a)

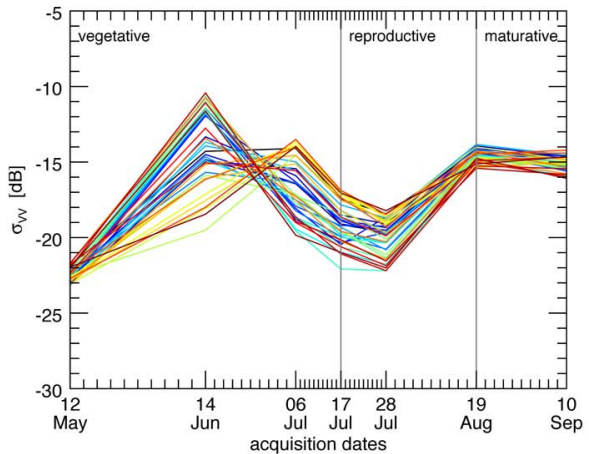

(b)

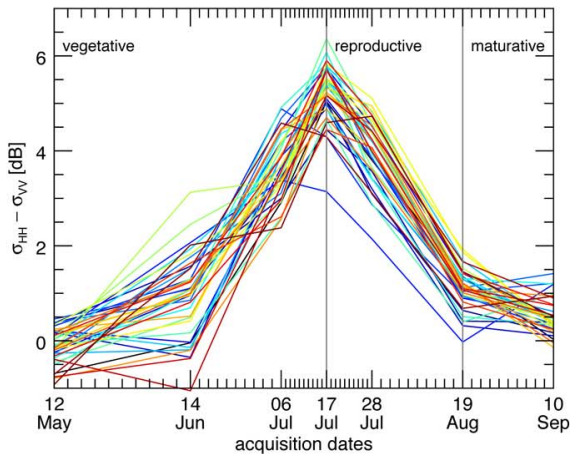

(c)

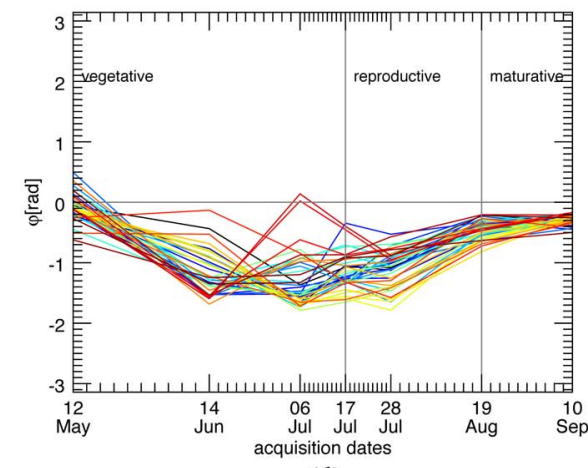

(d)

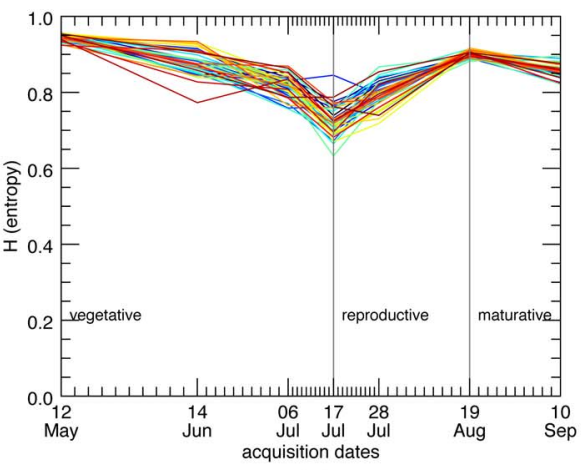

(e)

Fig. 3. Observed polarimetric features such as (a) $\sigma_{\mathrm{HH}}$, (b) $\sigma_{\mathrm{VV}}$, (c) $\sigma_{\mathrm{HH}}-\sigma_{\mathrm{VV}}$, (d) CPD, and (e) entropy through the entire cultivation period for 50 randomly selected neighbor paddy-rice fields. Each agricultural field appears in a different color. The plotted transition dates are estimated from time-series analysis.

The temporal behavior of the backscattering intensity, i.e., sigma nought, during the cultivation period is shown with the $\mathrm{HH}$ and VV polarizations in Fig. 3(a) and (b), respectively. Considering the low values of $\sigma_{\mathrm{HH}}$ and $\sigma_{\mathrm{vV}}$, we can infer that the fields are flooded in the beginning of May. Then, when the paddy elevation starts to be over the water level, the total backscattering coefficient increases, indicating the existence of unfolded leaves and the beginning of leaf development. The overall backscatter level is particularly high at this stage due to the strong presence of signal double bounces. A loss is then perceived by the cause of tillering, followed by panicle initiation. Here, the appearance of two distinct peaks corresponds to different grow rates for the chosen fields as a result of different agricultural practices (see also [8]). Whenever the canopy becomes tall enough and the panicle initiation starts, a decrease in the backscattering is observed for both channels due to higher absorption. It is to be noted that the VV channel [see Fig. 3(b)] exhibits a higher loss than the HH channel [see Fig. 3(a)], with the decay reaching almost the flooded field level of early May. The different extinction for the two polarizations in the presence of thin, fresh, and vertical structures is well noticeable here. Later on, the backscattering coefficients increase until the end of the reproductive stage (August 19). No evident discrepancy between the polarizations is any more visible.

The ratio between the two backscattering coefficients is plotted in Fig. 3(c). By considering this temporal aspect and the previous works in [1], [11], and [12], it is possible to assign the three main phenological stages of the canopy on the acquisition dates (vegetation, reproductive, maturation), as highlighted over the figures. In the study area, the reproductive stage started on July 17 for most of the fields, and the transition from the reproductive stage to the maturation stage began in late August. The assigned transition dates from the vegetative stage to the reproductive stage, which is one of the most critical events in a plant life, can be also proved by taking into account the temporal trend of the CPDs of the TanDEM-X acquisitions shown in Fig. 3(d). In the presence of standing water, straight after transplanting, the measured copolar phase $\varphi$ is around zero. This little depolarization implies, by definition, a smooth surface. The development of the standing erect canopy over the water surface then yields double-bounce scattering. This phenomenon causes a negative CPD. As the plant development continues further, the scattering becomes more homogeneous, and the vertical component diminishes. This yields a phase difference increase that is measured until the beginning of the maturation stage, which is around August 19 and the following harvesting. Due to the increased field homogeneity, the CPD variance gets lower as well. It may be noticed here that the differences in the seeding practice among the fields have resulted in different growth rates, which cause a high variance in the CPD values through the fields in the vegetative and reproductive stages. However, as the canopy becomes more mature, i.e., before harvesting, the path difference among the fields converges to null, and all the other polarimetric features of the agricultural fields turn out to be homogeneous.

Finally, the polarimetric decomposition proposed in [13] has been applied to evaluate the entropy parameter. This analysis provides information about temporal existing scattering mechanisms (a maximum of two) through the cultivation. The analysis of entropy given in Fig. 3(e) supports the assigned phenological step dates by the polarimetric features. Entropy, which characterizes the degree of randomness of the scattering, makes its minimum on July 17 , when the double bounce dominates other scattering types. Later, as the paddy continues to grow, 


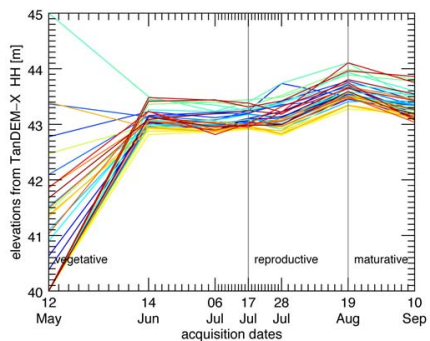

(a)

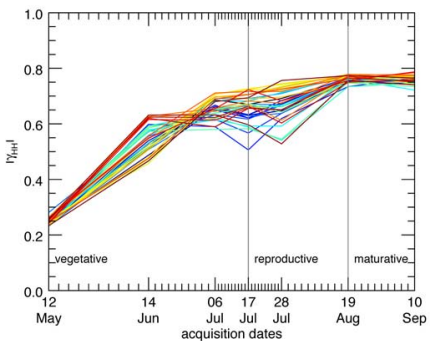

(c)

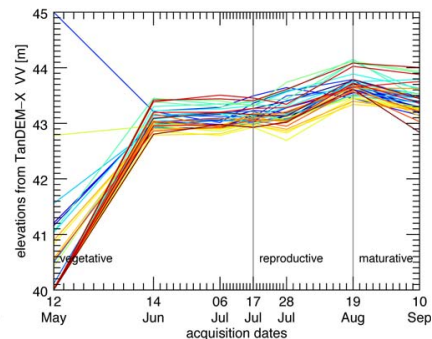

(b)

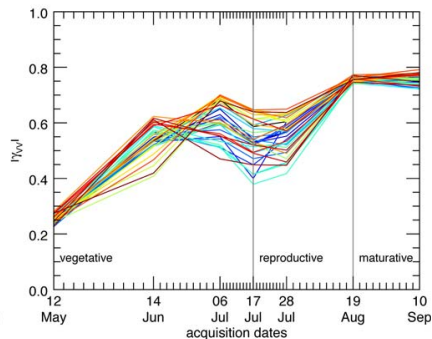

(d)
Fig. 4. Inventory of topographic surface changes. The value of multitemporal elevation measurements from the TanDEM-X HH and VV channels are shown in (a) and (b), respectively, and their reliability information is based on their coherence values shown in (c) and (d), respectively. The plotted transition dates are estimated from the time-series analysis.

the increase in randomness is observed again due to the higher intensiveness of the canopy. Here, it should be noticed that the high entropy at the beginning of the time series is due to the random process of the system noise, not to the presence of more than one dominant scattering. The flooded fields in May exhibit backscatter values that are very close to the system's noiseequivalent sigma zero, which is about $-23 \mathrm{~dB}$ for the beam under study.

\section{DEM ANALYSIS}

The features shown in the previous section demonstrate the possibility of estimating the phenological stage of the fields from copolarimetric TanDEM-X signatures where no ground measurements exist. In order to analyze the potential of height measurements using copolarimetric TanDEM-X data, these features will be useful in characterizing the height differences obtained from the HH- and VV-polarized interferograms. The analysis in the previous section has been performed for the monostatic channel of the TanDEM-X acquisition; in this section, the bistatic channel is used to generate the canopy elevation model.

The bistatic HH-polarized TanDEM-X data have been shown to be capable of tracking elevation changes at the decimetric level [8]. This study is expanded to explore the suitability of the VV polarization. To conduct this study, in Fig. 4, the elevation estimations are analyzed for the same fields shown in Fig. 3. Both the HH-polarized [see Fig. 4(a)] and VV-polarized [see Fig. 4(b)] TanDEM-X data give very similar temporal trends, with a maximum slope gradient in the reproductive stage. Although the height gradients of the agricultural fields coincide with the phenological stages of the canopy, the mean elevations on the acquisition on May 12 are found to be random for both polarizations. This result agrees with the mean coherence value $(\sim 0.25)$ and the mean sigma-nought value $(\sim-23 \mathrm{~dB})$ on that acquisition day, indicating flooded fields.
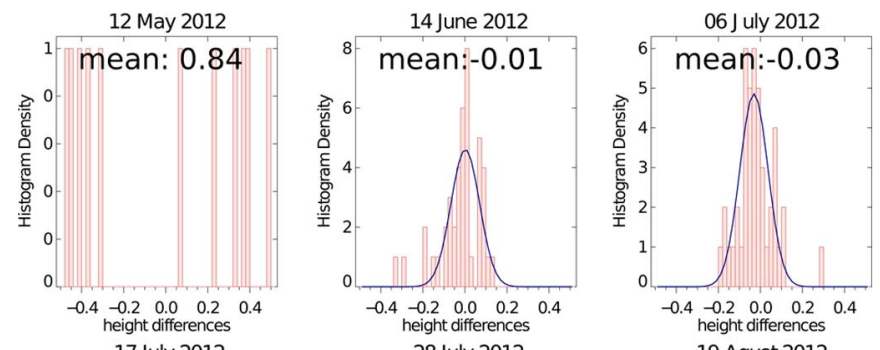

17 July 2012

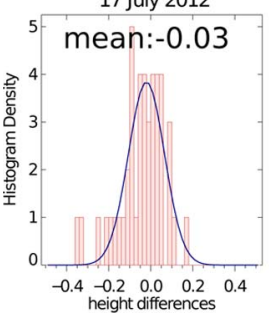

28 July 2012

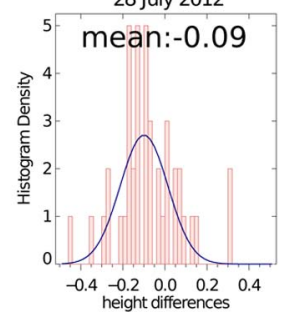

19 Agust 2012

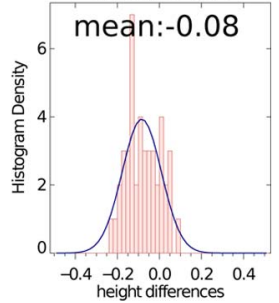

10 September 2012
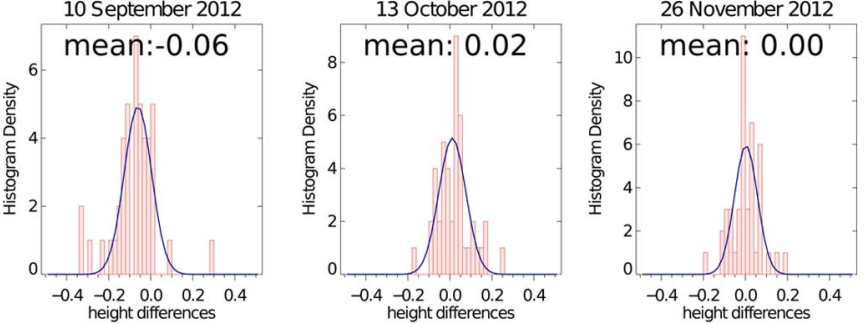

Fig. 5. Temporal histograms of the difference between the elevation measurements from the HH- and VV-polarized TanDEM-X images among the neighbor paddy fields. A Gaussian fit (blue line) is superimposed.

The sensitivity of the TanDEM-X acquisitions is assessed through the visual and qualitative comparison of the temporal CPD [see Fig. 3(d)] and the interferometric coherence values of the TanDEM-X measurements at the $\mathrm{HH}$ and VV acquisitions (see Fig. 4(c) and (d), respectively).

An evident visual divergence is for the late vegetative stage and the early reproductive stage (around July 17). Here, the $\mathrm{HH}$ elevation accuracy is larger than the VV elevation accuracy, as the coherence values are higher. The standard deviation is also smaller for the horizontal polarization. Thus, when considering assessing the crop elevation with bistatic data for the central growing stage, it seems meaningful to privilege the $\mathrm{HH}$ channel. By inspecting the CPD and considering the analysis in the previous section, it results in the VV channel yielding higher phase centers and therefore better modeling the top of the canopy. A choice is then difficult; a quantitative evaluation is provided in the following. On the contrary, the two other stages perform similarly, i.e., the early vegetative stage has very low coherence and poor elevation estimates for both channels, whereas the late reproductive and maturation stages perform well.

To make the polarization dissimilarity, i.e., distributing from the end of the vegetative stage to the beginning of the maturation stage, clearer, the probability density functions (pdfs) in terms of the histogram of the elevation differences are temporally plotted in Fig. 5. The histograms of the elevation differences from the HH- and VV-polarized acquisitions were established among the selected fields for each acquisition day. On one hand, for the flooded fields, the copolar elevation difference measurement is uniformly distributed because of the unreliable phase information. On the other hand, for the other acquisitions, the elevation differences are closer to the Gaussian 
TABLE I

SAMPle And Gaussian-Fit Statistics of The DifFerence BETWEEN THE HH AND VV ELEVATIONS

\begin{tabular}{|c|c|c|c|c|}
\hline \multirow{2}{*}{ Dates } & \multicolumn{2}{|c|}{ sample statistics } & \multicolumn{2}{c|}{ Gaussian-fit statistics } \\
\cline { 2 - 5 } & mean $(\mathrm{m})$ & std $(\mathrm{m})$ & center $(\mathrm{m})$ & std $(\mathrm{m})$ \\
\hline 12 May & 0.84 & 2.04 & - & - \\
14 Jun. & -0.01 & 0.23 & 0.00 & 0.07 \\
06 Jul. & -0.03 & 0.08 & -0.03 & 0.07 \\
17 Jul. & -0.03 & 0.11 & -0.02 & 0.09 \\
28 Jul. & -0.09 & 0.19 & -0.10 & 0.11 \\
19 Agu. & -0.08 & 0.07 & -0.08 & 0.09 \\
10 Sep. & -0.05 & 0.10 & -0.04 & 0.07 \\
23 Oct. & 0.02 & 0.07 & 0.01 & 0.05 \\
26 Nov. & 0.00 & 0.06 & 0.00 & 0.04 \\
\hline
\end{tabular}

distribution, with the mean and the variance strongly dependent on the radar parameters and the canopy properties.

It is interesting to see the effect of the extinction coefficient in the vertical channel through almost all the phenological stages. The temporal mean difference measurements monotonically increase until the acquisition date of July 28 . After, they monotonically decrease until the paddy rice starts to collapse and lose its height and, hence, the vertical structure. Similar results are reported in [14] for another vertical-structured canopy maize with indoor experiments. For the pdf obtained from the agricultural fields acquired on November 26 when the fields were already harvested, there is almost ( $\sim$ the millimeter level $)$ no difference between the polarimetric TanDEM-X measurements.

In this pdf-based analysis, the estimate of the sample mean elevation differences is not the only interest. A consistency analysis among the neighbor paddy fields is also important, and it is given in Table I. The sample standard deviations for each acquisition date show the variability of the outcomes for each phenological stage. The values are nearly stable through the maturation stage, but in the vegetative and reproductive stages, they are relatively high also considering the differences in the growing rate. As reported in Table I, a Gaussian-fit analysis is superimposed for each acquisition date histogram. Temporal Gaussian standard deviations are in accordance with the signature of the sample standard deviations. The horizontal polarization yields DEMs with lower crop heights, i.e., up to about 10 -cm differences. The vertical polarization yields higher elevation models, i.e., close to the true top canopy elevation [8]. As aforementioned, the horizontal polarization provides, on average, more accurate elevation results for the central growing stage. Stepping back to the polarization choice, if the objective is the determination of the crop elevation, the local field coherence can be the final trigger.

\section{CONCLUSION}

The analysis showed in this letter has been intended to provide knowledge about the use of dual-polarization TanDEM$\mathrm{X}$ data for paddy-rice monitoring, which is in extension to the work in [8], by completely considering the wave polarization. Our results clearly indicate that, according to the phenological stage of the canopy, there are differences between the height measurements of the TanDEM-X HH and VV channels. These differences in the height of the scattering phase center depend on the different attenuations of the polarized channels, reaching $10 \mathrm{~cm}$ in the reproductive stage. This discrepancy has to be considered in the operational applications in which the canopy height is used as a state variable, e.g., for indirect yield estimation and biomass calculation methods. In these works, the plant height factor is served as a state variable to specify the morphological characterization, and its accuracy in the centimeter level is important, particularly for large-scale agricultural fields.

Although the entire analysis is only given for paddy rice, the results can be generalized for the canopies having a vertical structure and a similar dielectric constant behavior as paddy rice. Typical polarimetric features as the backscatter ratio, the CPD, entropy, etc., are also studied in the test case and demonstrated well in accordance with previous studies.

\section{ACKNOWLEDGMENT}

The authors would like to thank the German Aerospace Center (DLR) for providing the TanDEM-X data set.

\section{REFERENCES}

[1] A. Bouvet, T. Le Toan, and N. Lam-Dao, "Monitoring of the rice cropping system in the Mekong delta using ENVISAT/ASAR dual polarization data," IEEE Trans. Geosci. Remote Sens., vol. 47, no. 2, pp. 517-526, Feb. 2009.

[2] Y. Inoue, E. Sakaiya, and C. Wang, "Capability of C-band backscattering coefficients from high-resolution satellite SAR sensors to assess biophysical variables in paddy rice," Remote Sens. Environ., vol. 140, pp. 257-266, Jan. 2014.

[3] V. Kalogirou, P. Ferrazzoli, A. Deulla Vecchia, and M. Foumelis, "On the SAR backscatter of burned forests: A model-based study in C-band, over burned pine canopies," IEEE Trans. Geosci. Remote Sens., vol. 52, no. 10, pp. 6205-6215, Oct. 2014.

[4] M. E. Engdahl, M. Borgeaud, and M. Rast, "The use of ERS 1/2 Tandem interferometric coherence in the estimation of agricultural crop heights," IEEE Trans. Geosci. Remote Sens., vol. 39, no. 8, pp. 1799-1806, Aug. 2001.

[5] X. Blaes and P. Defourny, "Retrieving crop parameters based on Tandem ERS 1/2 interferometric coherence images," Remote Sens. Environ., vol. 88 , no. 4 , pp. 374-385, Dec. 2003.

[6] G. Krieger et al., "TanDEM-X: A satellite formation for high-resolution SAR interferometry," IEEE Trans. Geosci. Remote Sens., vol. 45, no. 11, pp. 3317-3341, Nov. 2007.

[7] C. Rossi, F. R. Gonzales, T. Fritz, N. Yague-Martinez, and M. Eineder, "TanDEM-X calibrated raw DEM generation," ISPRS J. Photogramm. Remote Sens., vol. 73, pp. 12-20, Sep. 2012.

[8] C. Rossi and E. Erten, "Paddy-rice monitoring using TanDEM-X" IEEE Trans. Geosci. Remote Sens., vol. 53, no. 2, pp. 900-909, Feb. 2015.

[9] S. Leinss, G. Parrella, and I. Hajnsek, "Snow height determination by polarimetric phase differences in X-band SAR data," IEEE J. Sel. Topics Appl. Observ. Remote Sens., vol. 7, no. 9, pp. 3794-3810, Sep. 2014.

[10] O. Yüzügüllü, E. Erten, and I. Hajnsek, "Rice growth monitoring by means of X-band co-polar SAR: Feature clustering and BBCH scale," IEEE Geosci. Remote Sens. Lett., vol. 12, no. 6, pp. 1218-1222, Jun. 2015.

[11] J. M. Lopez-Sanchez, J. D. Ballester-Berman, and I. Hajnsek, "First results of rice monitoring practices in Spain by means of time series of TerraSAR-X dual-pol images," IEEE J. Sel. Topics Appl. Earth Observ. Remote Sens., vol. 4, no. 2, pp. 412-422, Jun. 2011.

[12] J. M. Lopez-Sanchez, S. R. Cloude, and J. D. Ballester-Berman, "Rice phenology monitoring by means of SAR polarimetry at X-band," IEEE Trans. Geosci. Remote Sens., vol. 50, no. 7, pp. 2695-2709, Jul. 2012.

[13] S. R. Cloude, "The dual polarization entropy/alpha decomposition: A PALSAR case study," in Proc. 3rd PolInSAR Workshop, Frascati, Italy, 2007, pp. 1-6.

[14] J. M. Lopez-Sanchez, J. Fortuny-Guasch, and J. D. Ballester-Berman, "Indoor wide-band polarimetric measurements on maize plants: A study of the differential extinction coefficient," IEEE Trans. Geosci. Remote Sens., vol. 4, no. 44, pp. 758-767, Apr. 2006. 